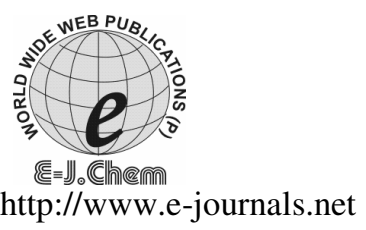

\title{
Microwave Assisted Synthesis of Some Biologically Active Benzothiazolotriazine Derivatives
}

\author{
PRASHANT KRIPLANI, PAWAN SWARNKAR, \\ RINKU MAHESHWARI and K.G.OJHA* \\ Department of Pure and Applied Chemistry \\ M. D. S. University Ajmer-305 009(India) \\ e-mail: kg_chemistry@ rediffmail.com
}

Received 20 July 2006; Accepted 22 August 2006

\begin{abstract}
Synthesis of some biologically active benzothiazolotriazine derivatives by microwave irradiation is reported. 2-Amino-6-substituted benzothiazoles 1 on treatment with benzaldehyde in anhydrous ethanol afforded 2benzylidenoimino-6-substitutedbenzothiazoles 2 which underwent cyclisation with ammoniumthiocyanate in dioxane to give 2-phenyl benzothiazolo [3,2- $\alpha]-\mathrm{s}-$ triazine-4-[3H] thiones 3.These both steps were carried out in microwave. Compound $\mathbf{3}$ with benzoyl chloride in anhydrous pyridine gave 2-phenyl-3(benzoyl) benzothiazolo [3,2- $\alpha$ ]-s-triazine-4-thiones 4 in good yields. The structure of all these compounds have been supported by their elemental analysis and their spectral data. All synthesized compounds were tested for their antibacterial activity using standard drug.
\end{abstract}

Keywords: Benzothiazoles, s-triazines, microwave irradiation, antibacterials

\section{Introduction}

Heterocyclic compounds containing nitrogen and sulphur possess potential pharmacological activities ${ }^{1-4}$. Benzothiazole moiety constitute an important class of heterocyclic compounds possessing diverse type of biological activities viz. antibacterial ${ }^{5}$, fungicidal ${ }^{6}$, antituberculotic ${ }^{7}$, antiallergic ${ }^{8}$, anticancer ${ }^{9}$ etc. Triazine derivatives are also associated with broad spectrum antibacterial ${ }^{10}$, antifungal ${ }^{11}$, antiviral activity ${ }^{12-14}$ against numerous viruses viz. Rauscher viruses, Leukemia Moloney viruses, Leukemia Rhinovirus type-2, influenza virus type-2,Vaccinia viruses, Vasicular stomatitis and Measules viruses. In view of the activities exhibit by benzothiazoles and triazines, we have reported synthesis of some new 
benzothiazolotriazine derivatives by conventional method in our earlier paper ${ }^{15}$.As a part of our continuing interest in biologically active benzothiazolotriazine derivatives, we are reporting a route for synthesis of these compounds by microwave irradiation. Traditional synthesis of compounds suffered from the disadvantages such as long reaction time, low yield and inconvenience of handling. In recent years the use of microwave technology in organic synthesis has received considerable attention. This technology can increase the purity of products, enhance the chemical yield and shorten the reaction time ${ }^{16}$. All synthesized compounds were tested for their antibacterial activity using standard drug.

\section{Experimental}

All the melting points are uncorrected. The purity of synthesized compounds has been checked by thin layer chromatography. IR spectra are recorded on FT-IR Perkin-Elmer (Spectrum RX1) spectrophotometer $\left(v_{\max }\right.$ in $\left.\mathrm{cm}^{-1}\right)$ using $\mathrm{KBr}$ disc. ${ }^{1} \mathrm{H}$ NMR spectra are recorded in $\mathrm{CDCl}_{3}$ on a Bruker DRX-300 MHz using TMS as internal standard. The chemical shifts are reported as parts per million (ppm).Microwave synthesis was carried out in a domestic microwave oven model L.G. MS-194W, 230-50 Hz., 800W.

\section{Microwave synthesis of 2-benzylidenoimino-6-substitutedbenzothiazoles 2}

A mixture of 2-amino-6-substitutedbenzothiazole $1(0.001 \mathrm{~mol})$ and benzaldehyde $(0.001$ mol) in minimum quantity of anhydrous ethanol were taken in Erlen Meyer flask capped with a funnel placed in a microwave oven and irradiated at 160 Watt for 1 to 1.5 minutes. The reaction was monitered by silica gel TLC. (Benzene : Acetone $70: 30$ ).After completion the reaction, the reaction mixture was allowed to attain room temperature and solid separated was filtered. The crude product was recrystallized from redistilled ethanol.

Microwave synthesis of 2-phenyl benzothiazolo [3,2- $\alpha$ ]-s-triazine-4-[3H] thiones 3

A mixture of 2-benzylidenoimino-6-substitutedbenzothiazole 2 (0.001mol) and ammoniumthiocyanate $(0.002 \mathrm{~mol})$ were dissolved in minimum quantity of 1,4 -dioxane and were taken in Erlen Meyer flask capped with a funnel placed in a microwave oven and irradiated at 160 Watt for 1.5 to 2 minutes. The reaction was monitered by silica gel TLC.(Hexane : DMF $80: 20)$.After completion the reaction, the reaction mixture was allowed to attain room temperature and solid separated was filtered. The crude product was recrystallized from redistilled ethanol.

Synthesis of 2-phenyl-3-(benzoyl) benzothiazolo [3,2- $\alpha$ ]-s-triazine-4-thiones 4

2-Phenyl benzothiazolo [3,2- $\alpha]$-s-triazine-4-[3H] thiones $3(0.005 \mathrm{~mol})$ was dissolved in minimum quantity of anhydrous pyridine $(10 \mathrm{ml})$.To this solution was added benzoyl chloride $(0.01 \mathrm{~mol})$ dropwise with constant shaking in cold conditions. The reaction mixture was further stirred for 1 hour and poured into acidified icecold water. The solid separated out was filtered and washed repeatedly with water, dried in vacuo and recrystallised from redistilled ethanol.

Spectral Analysis of compounds $2 a-2 d, 3 a-3 d, 4 a-4 d$.

Compound 2a: M.F. $\mathrm{C}_{14} \mathrm{H}_{9} \mathrm{~N}_{2} \mathrm{SCl}$, IR (KBr) $v_{\max }$ in $\mathrm{cm}^{-1} 859(\mathrm{C}-\mathrm{Cl}), 1349(\mathrm{C}-\mathrm{S}), 1597(\mathrm{C}=\mathrm{N})$, $1447,1535(\mathrm{ArC}=\mathrm{C}),{ }^{1} \mathrm{HNMR}\left(300 \mathrm{MHz}, \mathrm{CDCl}_{3}\right): \delta 4.02(\mathrm{~s}, 1 \mathrm{H},=\mathrm{CHPh}), 7.10-7.62(\mathrm{~m}, 8 \mathrm{H}, \mathrm{ArH})$,

Compound 2b: M.F.C ${ }_{14} \mathrm{H}_{9} \mathrm{~N}_{2} \mathrm{SBr}$, IR (KBr) $v_{\max }$ in $\mathrm{cm}^{-1} 809(\mathrm{C}-\mathrm{Br}), 1376(\mathrm{C}-\mathrm{S}), 1598(\mathrm{C}=\mathrm{N})$, 1460,1531 $(\mathrm{ArC}=\mathrm{C}),{ }^{1} \mathrm{HNMR}\left(300 \mathrm{MHz}, \mathrm{CDCl}_{3}\right): \delta 4.0(\mathrm{~s}, 1 \mathrm{H},=\mathrm{CHPh}), 7.02-7.52(\mathrm{~m}, 8 \mathrm{H}, \mathrm{ArH})$, 
Compound 2c: M.F. $\mathrm{C}_{14} \mathrm{H}_{9} \mathrm{~N}_{3} \mathrm{SO}_{2}$, IR (KBr) $v_{\max }$ in $\mathrm{cm}^{-1} 1380,1510\left(-\mathrm{NO}_{2}\right), 1597(\mathrm{C}=\mathrm{N})$, 1346(C-S), 1436,1490,1539(ArC=C), ${ }^{1} \mathrm{HNMR}\left(300 \mathrm{MHz}, \mathrm{CDCl}_{3}\right): \delta 5.6(\mathrm{~s}, 1 \mathrm{H},=\mathrm{CHPh}), 7.20-$ 7.90(m,8H, ArH),

Compound 2d: M.F. $\mathrm{C}_{16} \mathrm{H}_{14} \mathrm{~N}_{2} \mathrm{SO}$, IR (KBr) $v_{\max } \mathrm{cm}^{-1} 1058$ (C-O-Csym.) 1209 (C-O-Casym.), 1457,1545(ArC=C), 1596(C=N), 2974(C-Hstr.), ${ }^{1} \mathrm{HNMR}\left(300 \mathrm{MHz}, \mathrm{CDCl}_{3}\right): \delta 1.45\left(\mathrm{t}, 3 \mathrm{H}_{1}, \mathrm{CH}_{3}\right)$, $4.0\left(\mathrm{q}, 2 \mathrm{H}, \mathrm{OCH}_{2}\right), 5.4(\mathrm{~s}, 1 \mathrm{H},=\mathrm{CHPh}), 7.25-7.55(\mathrm{ArH})$

Compound 3a: M.F. $\mathrm{C}_{15} \mathrm{H}_{10} \mathrm{~N}_{3} \mathrm{~S}_{2} \mathrm{Cl}$, IR (KBr) $v_{\max }$ in $\mathrm{cm}^{-1} 864(\mathrm{C}-\mathrm{Cl}), 1310(\mathrm{C}-\mathrm{S})$, $1580(\mathrm{C}=\mathrm{N}), 1440,1535(\mathrm{ArC}=\mathrm{C}), 3455(\mathrm{~N}-\mathrm{Hstr}),.{ }^{1} \mathrm{HNMR}\left(300 \mathrm{MHz}, \mathrm{CDCl}_{3}\right): \delta 5.5(\mathrm{~s}, 1 \mathrm{H},=\mathrm{CHPh})$, 3.6(s,1H,NH), 7.30-7.92(m,8H,ArH),

Compound 3b: M.F. $\mathrm{C}_{15} \mathrm{H}_{10} \mathrm{~N}_{3} \mathrm{~S}_{2} \mathrm{Br}$, IR (KBr) $v$ max in $\mathrm{cm}^{-1} 811(\mathrm{C}-\mathrm{Br}), 1350(\mathrm{C}-\mathrm{S})$, $1589(\mathrm{C}=\mathrm{N}), 1460,1531(\mathrm{ArC}=\mathrm{C}), 3400(\mathrm{~N}-\mathrm{Hstr}),{ }^{1} \mathrm{HNMR}\left(300 \mathrm{MHz}, \mathrm{CDCl}_{3}\right): \delta 5.0(\mathrm{~s}, 1 \mathrm{H},=\mathrm{CHPh})$, 3.8( $\mathrm{s}, 1 \mathrm{H}, \mathrm{NH}), 7.23-7.88(\mathrm{~m}, 8 \mathrm{H}, \mathrm{ArH})$,

Compound 3c: M.F. $\mathrm{C}_{15} \mathrm{H}_{10} \mathrm{~N}_{4} \mathrm{~S}_{2} \mathrm{O}_{2}$, IR (KBr) $v$ max in $\mathrm{cm}^{-1} 1385,1505\left(-\mathrm{NO}_{2}\right)$, $1580(\mathrm{C}=\mathrm{N}) 1330(\mathrm{C}-\mathrm{S}), \quad 1430-1539(\mathrm{ArC}=\mathrm{C}), 3450(\mathrm{~N}-\mathrm{Hstr}),{ }^{1} \mathrm{HNMR} \quad\left(300 \mathrm{MHz}, \mathrm{CDCl}_{3}\right): \delta$ $4.5(\mathrm{~s}, 1 \mathrm{H},=\mathrm{CHPh}), 3.8(\mathrm{~s}, 1 \mathrm{H}, \mathrm{NH}), 7.50-7.90(\mathrm{~m}, 8 \mathrm{H}, \mathrm{ArH})$,

Compound 3d: M.F. $\mathrm{C}_{17} \mathrm{H}_{15} \mathrm{~N}_{3} \mathrm{~S}_{2} \mathrm{O}$, IR (KBr) $v_{\max }$ in $\mathrm{cm}^{-1}$ 1054(C-O-Csym.)1225(C-O-Casym.), 1465,1540(ArC=C), $1596(\mathrm{C}=\mathrm{N}), \quad 2860\left(\mathrm{C}-\mathrm{Hstr}\right.$ ), 3305(N-Hstr.), ${ }^{1} \mathrm{HNMR} \quad\left(300 \mathrm{MHz}, \mathrm{CDCl}_{3}\right)$ : $\delta 1.3\left(\mathrm{t}, 3 \mathrm{H}, \mathrm{CH}_{3}\right), 4.2\left(\mathrm{q}, 2 \mathrm{H}, \mathrm{OCH}_{2}\right), 6.5(\mathrm{~s}, 1 \mathrm{H},=\mathrm{CHPh}), 7.28-8.00(\mathrm{~m}, 8 \mathrm{H}, \mathrm{ArH}), 3.8(\mathrm{~s}, 1 \mathrm{H}, \mathrm{NH})$,

Compound 4a : M.F. $\mathrm{C}_{22} \mathrm{H}_{14} \mathrm{~N}_{3} \mathrm{~S}_{2} \mathrm{Cl}$, IR (KBr) $v$ max in $\mathrm{cm}^{-1} 856(\mathrm{C}-\mathrm{Cl}), 1340(\mathrm{C}-\mathrm{S})$, $1590(\mathrm{C}=\mathrm{N}), \quad 1440-1545(\mathrm{ArC}=\mathrm{C}), 1629(\mathrm{C}=\mathrm{O}), \quad{ }^{1} \mathrm{HNMR} \quad\left(300 \mathrm{MHz}, \mathrm{CDCl}_{3}+\mathrm{DMSO}_{6}\right): \delta$ $6.4(\mathrm{~s}, 1 \mathrm{H},=\mathrm{CHPh}), 7.6-8.14(\mathrm{~m}, 13 \mathrm{H}, \mathrm{ArH})$,

Compound 4b : M.F. $\mathrm{C}_{22} \mathrm{H}_{14} \mathrm{~N}_{3} \mathrm{~S}_{2} \mathrm{Br}$, IR (KBr) $v$ max in $\mathrm{cm}^{-1} 810(\mathrm{C}-\mathrm{Br}), 1349(\mathrm{C}-\mathrm{S})$, $1589(\mathrm{C}=\mathrm{N}), \quad 1435-1535(\mathrm{ArC}=\mathrm{C}), 1650(\mathrm{C}=\mathrm{O}),{ }^{1} \mathrm{HNMR} \quad\left(300 \mathrm{MHz}, \mathrm{CDCl}_{3}+\mathrm{DMSO}-\mathrm{d}_{6}\right): \delta$ $5.9(\mathrm{~s}, 1 \mathrm{H},=\mathrm{CHPh}), 7.50-8.00(\mathrm{~m}, 13 \mathrm{H}, \mathrm{ArH})$,

Compound 4c : M.F. $\mathrm{C}_{22} \mathrm{H}_{14} \mathrm{~N}_{4} \mathrm{~S}_{2} \mathrm{O}_{2}$, IR (KBr) $v_{\max }$ in $\mathrm{cm}^{-1} 1380,1505$ (-NO2), 1585 $(\mathrm{C}=\mathrm{N}) 1335(\mathrm{C}-\mathrm{S}), 1439-1548(\mathrm{ArC}=\mathrm{C}), 1640(\mathrm{C}=\mathrm{O}),{ }^{1} \mathrm{HNMR}\left(300 \mathrm{MHz}, \mathrm{CDCl}_{3}+\mathrm{DMSO}^{-\mathrm{d}_{6}}\right): \delta$ $6.0(\mathrm{~s}, 1 \mathrm{H},=\mathrm{CHPh}), 7.55-8.15(\mathrm{~m}, 13 \mathrm{H}, \mathrm{ArH})$,

Compound 4d : M.F. $\mathrm{C}_{24} \mathrm{H}_{19} \mathrm{~N}_{3} \mathrm{~S}_{2} \mathrm{O}$, IR (KBr) $v_{\max }$ in $\mathrm{cm}^{-1} 1040(\mathrm{C}-\mathrm{O}-\mathrm{Csym}$.) $1220(\mathrm{C}-$ O-Casym.), 1440-1550(ArC=C),1596(C=N),1650(C=O),2860(C-Hstr.), ${ }^{1} \mathrm{HNMR} \quad(300 \mathrm{MHz}$, $\left.\mathrm{CDCl}_{3}+\mathrm{DMSO}_{-} \mathrm{d}_{6}\right): \delta 1.45\left(\mathrm{t}, 3 \mathrm{H}, \mathrm{CH}_{3}\right), 4.1\left(\mathrm{q}, 2 \mathrm{H}, \mathrm{OCH}_{2}\right), 5.7(\mathrm{~s}, 1 \mathrm{H},=\mathrm{CHPh}), 7.28-8.00(\mathrm{~m}, 13 \mathrm{H}, \mathrm{ArH})$.

\section{Results and Discussion}

The required 2-amino-6-substitutedbenzothiazoles 1 were prepared by methods reported in literature ${ }^{17,18}$. The synthesis of compounds $\mathbf{3}$ starting from 2-amino-6-substituted benzothiazoles 1 from conventional method was reported earlier ${ }^{15}$ by us. The same reaction scheme was carried out under microwave conditions. It is noteworthy that the reaction which required 4 to 6 hours in conventional methods, was completed within 1 to 2 minutes under microwave conditions and yields have also been improved. Finally compounds $\mathbf{3}$ on treatment with benzoyl chloride in presence of anhydrous pyridine in acidified cold conditions gave compounds 4(Figure 1). All the synthesized compounds have been characterized on the basis of their physico-chemical data (Table 1) and spectral analysis. Compounds 3 and $\mathbf{4}$ contain chiral centre and thus exhibit optical activity. The products obtained after purification are dextrorotatory as observed by their optical activity in acetone solution. It seems that the laevo products are obtained in minor quantities and are removed during purification and crystallization. 


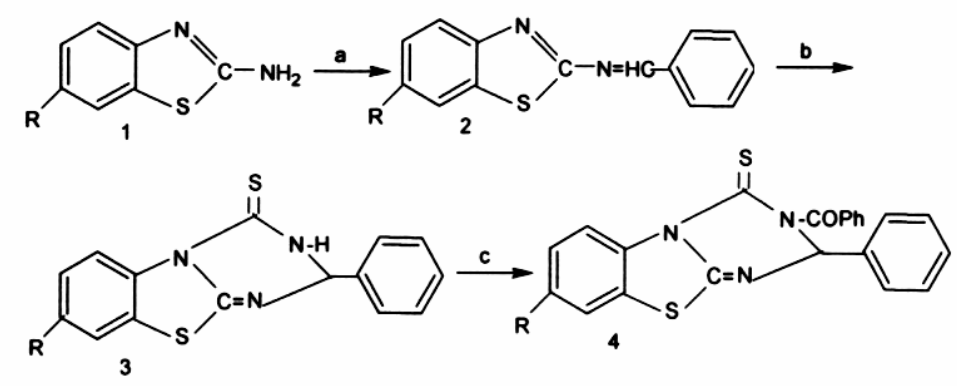

Figure 1. Reagents and Conditions : (a) Benzaldehyde, Ethyl alcohol, MWI for 1-1.5 minutes (b) $\mathrm{NH}_{4} \mathrm{SCN}, 1,4-$ dioxane, MWI for 1.5-2.0 minutes (c) PhCOCl, Pyridine, in cold conditions.

Table 1. Physico-Chemical data of synthesized compounds (C.M.=Conventional method, M.W.=Microwave)

\begin{tabular}{|c|c|c|c|c|c|c|c|c|c|}
\hline \multirow{3}{*}{$\begin{array}{l}\bar{\Xi} \\
\bar{\Xi} \\
\stackrel{0}{\Xi} \\
\text { 己े }\end{array}$} & \multirow[b]{3}{*}{$\mathrm{R}$} & \multirow{2}{*}{\multicolumn{2}{|c|}{$\begin{array}{l}\text { Reaction } \\
\text { Period }\end{array}$}} & \multirow{2}{*}{\multicolumn{2}{|c|}{$\begin{array}{c}\text { Yield } \\
\%\end{array}$}} & \multirow{3}{*}{$\begin{array}{c}\text { M.P. } \\
{ }^{0} \mathrm{C} \\
\text { M.W } \\
\text { (C.M.) }\end{array}$} & \multirow{2}{*}{\multicolumn{3}{|c|}{$\begin{array}{l}\text { Elemental Analysis } \\
\text { Cald } /(\text { Found }) \%\end{array}$}} \\
\hline & & & & & & & & & \\
\hline & & $\begin{array}{c}\text { C.M } \\
\mathrm{h}\end{array}$ & $\begin{array}{l}\text { M.W } \\
\text { min }\end{array}$ & C.M & M.W & & $\mathrm{C}$ & $\mathrm{H}$ & $\mathrm{N}$ \\
\hline $2 \mathrm{a}$ & $\mathrm{Cl}$ & $4^{15}$ & 1 & $66^{15}$ & 85 & $\begin{array}{c}161 \\
(160)^{15}\end{array}$ & $\begin{array}{c}61.05 \\
(60.09)\end{array}$ & $\begin{array}{c}0.03 \\
(0.028)\end{array}$ & $\begin{array}{c}10.27 \\
(11.11)\end{array}$ \\
\hline $2 b$ & $\mathrm{Br}$ & $4^{15}$ & 1 & $60^{15}$ & 90 & $\begin{array}{c}190 \\
(190)^{15}\end{array}$ & $\begin{array}{c}52.99 \\
(51.96)\end{array}$ & $\begin{array}{c}0.02 \\
(0.018)\end{array}$ & $\begin{array}{c}8.83 \\
(7.98)\end{array}$ \\
\hline $2 c$ & $\mathrm{NO}_{2}$ & $4^{15}$ & 1.5 & $58^{15}$ & 80 & $\begin{array}{c}262 \\
(260)^{15}\end{array}$ & $\begin{array}{c}59.36 \\
(59.23)\end{array}$ & $\begin{array}{c}0.03 \\
(0.028)\end{array}$ & $\begin{array}{c}14.84 \\
(15.10)\end{array}$ \\
\hline $2 \mathrm{~d}$ & $\mathrm{OC}_{2} \mathrm{H}_{5}$ & $4^{15}$ & 1.5 & $75^{15}$ & 95 & $\begin{array}{c}120 \\
(120)^{15}\end{array}$ & $\begin{array}{c}68.08 \\
(68.01)\end{array}$ & $\begin{array}{c}0.04 \\
(0.041)\end{array}$ & $\begin{array}{c}9.92 \\
(9.98)\end{array}$ \\
\hline $3 a$ & $\mathrm{Cl}$ & $6^{15}$ & 2 & $70^{15}$ & 88 & $\begin{array}{c}111 \\
(110)^{15}\end{array}$ & $\begin{array}{c}54.29 \\
(55.54)\end{array}$ & $\begin{array}{c}0.03 \\
(0.027)\end{array}$ & $\begin{array}{l}12.66 \\
(13.48)\end{array}$ \\
\hline $3 b$ & $\mathrm{Br}$ & $6^{15}$ & 1.5 & $55^{15}$ & 70 & $\begin{array}{c}126 \\
(125)^{15}\end{array}$ & $\begin{array}{c}47.87 \\
(47.79)\end{array}$ & $\begin{array}{c}0.02 \\
(0.018)\end{array}$ & $\begin{array}{c}11.17 \\
(11.14)\end{array}$ \\
\hline $3 c$ & $\mathrm{NO}_{2}$ & $6^{15}$ & 2 & $49^{15}$ & 66 & $\begin{array}{c}285 \\
(285)^{15}\end{array}$ & $\begin{array}{c}52.63 \\
(51.99)\end{array}$ & $\begin{array}{c}0.03 \\
(0.029)\end{array}$ & $\begin{array}{c}16.37 \\
(16.23)\end{array}$ \\
\hline $3 d$ & $\mathrm{OC}_{2} \mathrm{H}_{5}$ & $6^{15}$ & 2 & $68^{15}$ & 90 & $\begin{array}{l}166 \\
(165)^{15}\end{array}$ & $\begin{array}{c}59.82 \\
(56.88)\end{array}$ & $\begin{array}{c}0.04 \\
(0.039)\end{array}$ & $\begin{array}{l}12.31 \\
(12.34)\end{array}$ \\
\hline $4 a$ & $\mathrm{Cl}$ & - & - & $63^{15}$ & & $\begin{array}{c}136 \\
(136)^{15}\end{array}$ & $\begin{array}{c}62.93 \\
(61.74)\end{array}$ & $\begin{array}{c}0.03 \\
(0.04)\end{array}$ & $\begin{array}{l}10.01 \\
(9.97)\end{array}$ \\
\hline $4 b$ & $\mathrm{Br}$ & - & - & $46^{15}$ & & $\begin{array}{c}105 \\
(105)^{15}\end{array}$ & $\begin{array}{c}56.89 \\
(55.67)\end{array}$ & $\begin{array}{c}0.03 \\
(0.026)\end{array}$ & $\begin{array}{l}9.05 \\
(9.10)\end{array}$ \\
\hline $4 c$ & $\mathrm{NO}_{2}$ & - & - & $55^{15}$ & & $\begin{array}{c}300 \\
(298)^{15}\end{array}$ & $\begin{array}{c}61.39 \\
(60.38)\end{array}$ & $\begin{array}{c}0.03 \\
(0.031)\end{array}$ & $\begin{array}{c}13.02 \\
(13.00)\end{array}$ \\
\hline $4 d$ & $\mathrm{OC}_{2} \mathrm{H}_{5}$ & & & $70^{15}$ & & $\begin{array}{c}130 \\
(130)^{15} \\
\end{array}$ & $\begin{array}{c}67.13 \\
(66.78) \\
\end{array}$ & $\begin{array}{c}0.04 \\
(0.034) \\
\end{array}$ & $\begin{array}{c}9.79 \\
(9.78) \\
\end{array}$ \\
\hline
\end{tabular}




\section{Antibacterial Activity}

All the synthesized compounds were screened for their antibacterial activity against E.Coli, Pseudomonas aeruginosa and Staphylococcus aureus using Muller Hinton Agar media (Hi Media). The activity was carried out using paper disc method. The zone of inhibition measured in $\mathrm{mm}$. The results of antibacterial activity were tabulated (Table 2) in the form of activity index.

Table 2 . Antibacterial activity of synthesized compounds

\begin{tabular}{ccccc}
\hline S.No. & Compd. & E.Coli & Ps.aeruginosa & S. aureus \\
\hline 1 & $2 \mathrm{a}$ & 0.93 & 0.68 & 1.11 \\
2 & $2 \mathrm{~b}$ & 1.00 & 1.00 & 1.39 \\
3 & $2 \mathrm{c}$ & 1.16 & 1.20 & 1.66 \\
4 & $2 \mathrm{~d}$ & 0.80 & 0.84 & 1.22 \\
5 & $3 \mathrm{a}$ & 1.00 & 0.84 & 1.33 \\
6 & $3 \mathrm{~b}$ & 1.06 & 1.12 & 1.66 \\
7 & $3 \mathrm{c}$ & 1.16 & 1.28 & 1.89 \\
8 & $3 \mathrm{~d}$ & 1.00 & 1.04 & 1.61 \\
9 & $4 \mathrm{a}$ & 1.03 & 0.92 & 1.50 \\
10 & $4 \mathrm{~b}$ & 1.30 & 1.44 & 1.94 \\
11 & $4 \mathrm{c}$ & 1.33 & 1.40 & 2.11 \\
12 & $4 \mathrm{~d}$ & 1.00 & 1.24 & 1.72 \\
13 & Ceftazidime & 1.00 & 1.00 & 1.00 \\
\hline
\end{tabular}

\section{Activity index $=\frac{\text { Zone of inhibition of compound in } \mathbf{~ m m}}{\text { Zone of inhibition of standard drug in } \mathbf{~ m m}}$}

DMF was used as a solvent. Standard drug Ceftazidime $(\mathrm{Ca})(30 \mu \mathrm{g} / \mathrm{ml})$ was used for comparison. The compounds were tested at $500 \mu \mathrm{g} / \mathrm{ml}$ concentration. The observations show that activity index of compound $\mathbf{4 c}$ is maximum against E.Coli, activity index of compound $\mathbf{4 b}$ is maximum against Pseudomonas aeruginosa, activity index of compound $\mathbf{4 c}$ is maximum against Staphylococcus aureus.

\section{Conclusion}

In above synthetic scheme we use microwave irradiation technique, this leads to considerable saving in the reaction time and energetically profitable. The smaller volume of solvent required contributes to saving in cost and diminishes the waste disposal problem. Compounds $4 \mathrm{~b}$ and $4 \mathrm{c}$ show potential antibacterial activity.

\section{Acknowledgement}

Authors are thankful to Head, Department of Pure and Applied Chemistry, M.D.S University, Ajmer India for providing necessary laboratory facilities, to the Director, 
CDRI Lucknow India for providing elemental analysis, spectral analysis and Head, Department of Microbiology, J.L.N. Medical College, Ajmer India for providing antibactrial screening facility. Authors also express thanks to CSIR New Delhi India for providing JRF to one of them.(P. Kriplani)

\section{References}

1. Katrizky A R,”Advances in Heterocylic Chemistry” Academic Press, London $1985,135$.

2. Proto G and Thomson R H, Endeavour 1976,35, 32.

3. Faria C, Pinza M, Gabma A and Piffen G, Eur.J.Med. Chem.Chim.Ther., 1979,14, 27.

4. Roberts J J and Warwich G P, Biochem. Pharmacol., 1963,12,135.

5. Ansari A S and Banerji J C B, J. Ind. Chem. Soc., 1998,75,108.

6. Sidoova E and Bujdakova H, Pharmazie., 1994,49,375.

7. Waisser K, Dolezal M, Sidoova. E and Odlerova Z, Drasta. J., Chem.Abstr., 1989,110,128063e

8. Uclaf Rousel, Kokai Jpn and Koho, Tokkyo., Chem. Abstr., 1987,106,15649g.

9. Wells G, Bradshaw T D, Diana P, Seaton A, Shi D F, Westwell A D and Stevens M F G, Bioorg. \& Med.Chem.lett., 2000,10, 513.

10. Joshua C P, George Abraham and Alaudeen M, J. Indian Chem.Soc., 2004,81,357.

11. Mohan J and Anupama, Indian J.Chem., 2003,42B,2003.

12. Poonian M S, Nowoswiat E F, Blount J F and Karmer M J, J. Med.Chem. 1976,19,1017

13. Misra V S, Dhar S, Chowdhary B L, Pharmazie, 1976,33 ,790.

14. Chirigos M A, Moloney J B, Humphreys S R, Mantle N, Goldin A, Cancer Res., 1961,21,803.

15. Kriplani P, Swarnkar P and Ojha K G, Heterocyclic Communications 2005, 11(6), 527.

16. (a) Galena S A, Chem.Soc. Rev., 1997,26,233. (b) Sonali R ,Resonance, 2000,5,77.

(c) Lindstrom P, Tierney J, Wathey B and Westman J,Tetrahedron,2001,57,9225.

(d). More D H, Pawar. N S, Dewang. P M, Patil S L, Mahulikar P P, Rus .J

Gen. Chem, 2001, 74(2), 2244. (e) More D H, Pawar N S, Mahulikar P P, J. Sci. Ind.Res. 2003,62,1024.

17. Gupta R R, Jain S K and Ojha K G, Synth.Commun., 1979,9(6), 457.

18. Gupta R R, Ojha K G, Kumar M, J Heterocyclic Chem., 1980, 17,1325. 


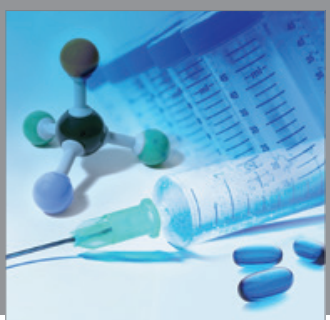

International Journal of

Medicinal Chemistry

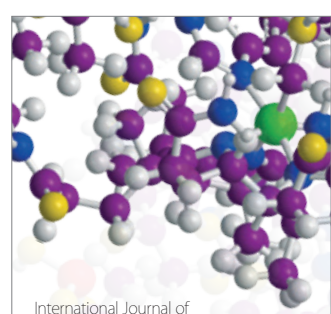

Carbohydrate Chemistry

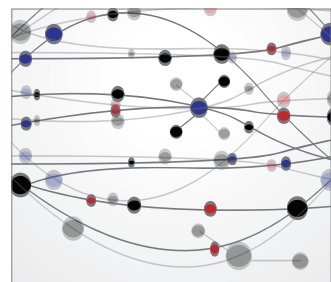

The Scientific World Journal
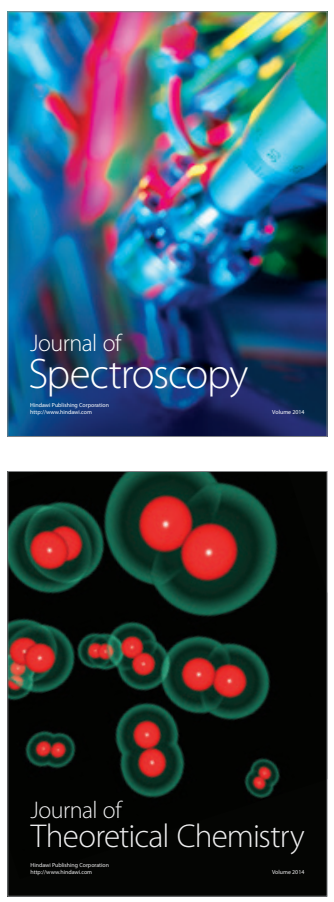
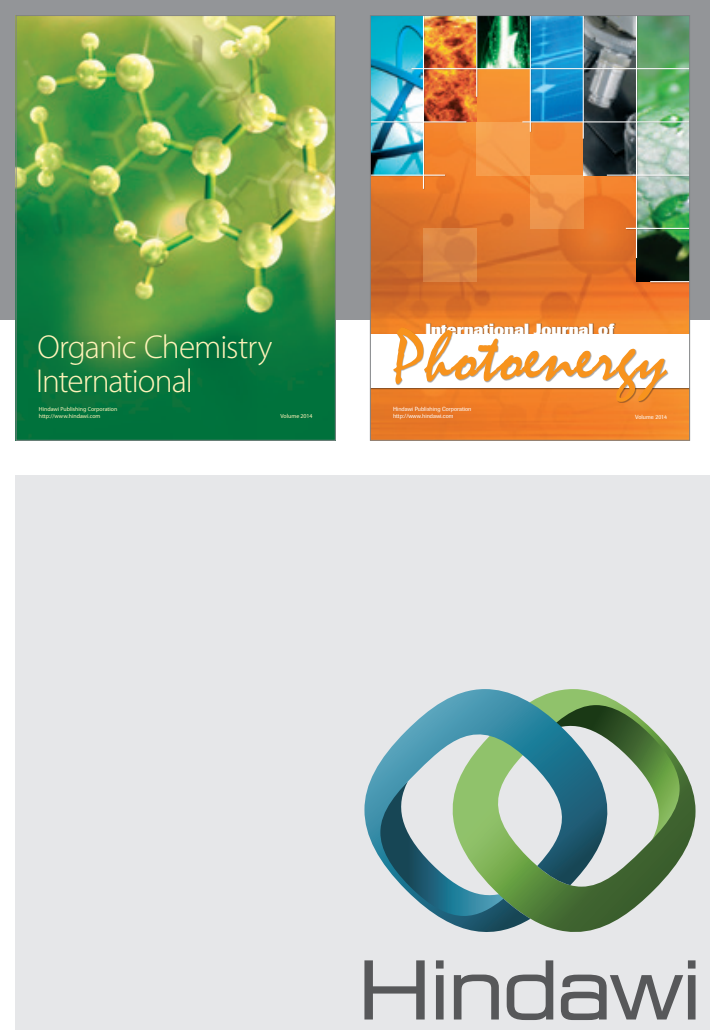

Submit your manuscripts at

http://www.hindawi.com
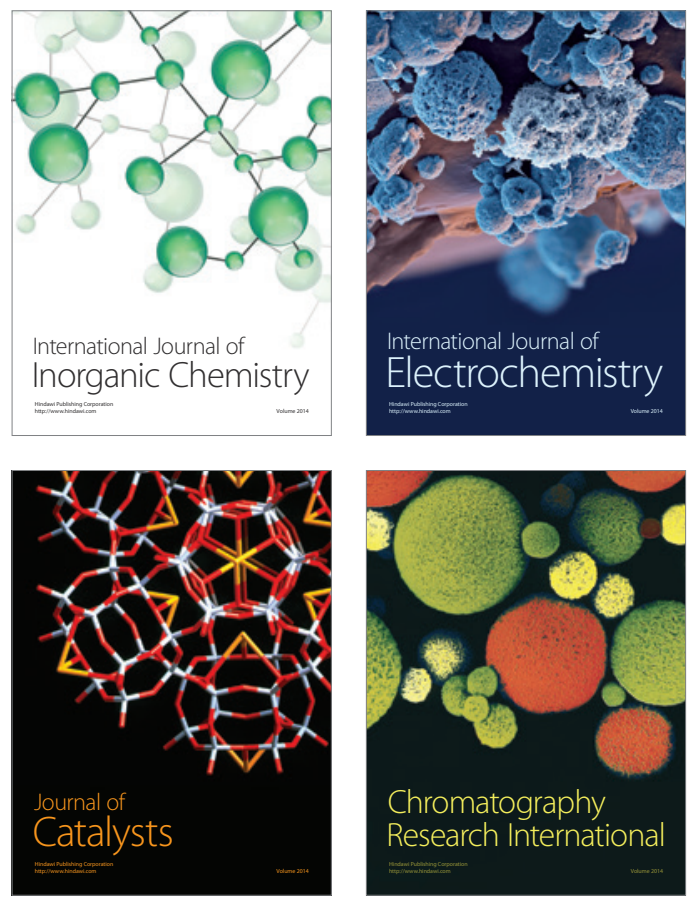
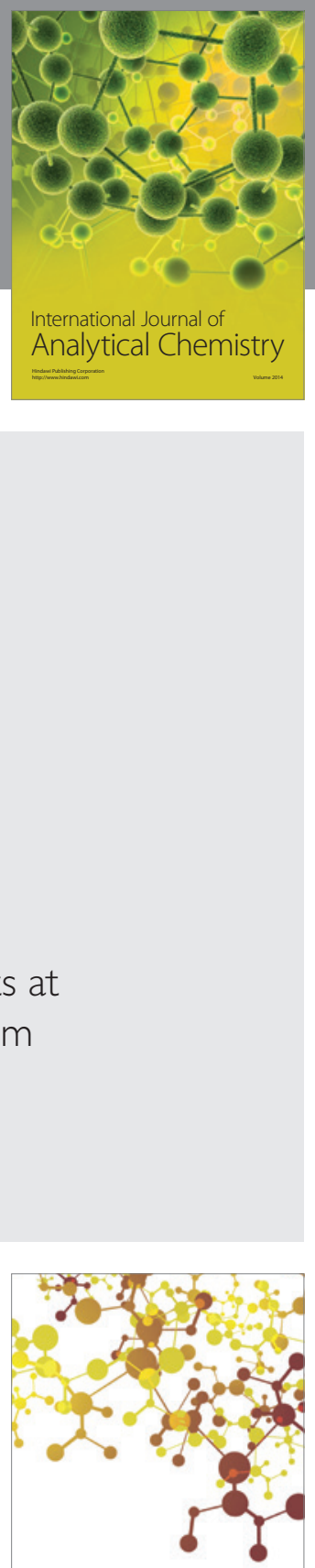

Journal of

Applied Chemistry
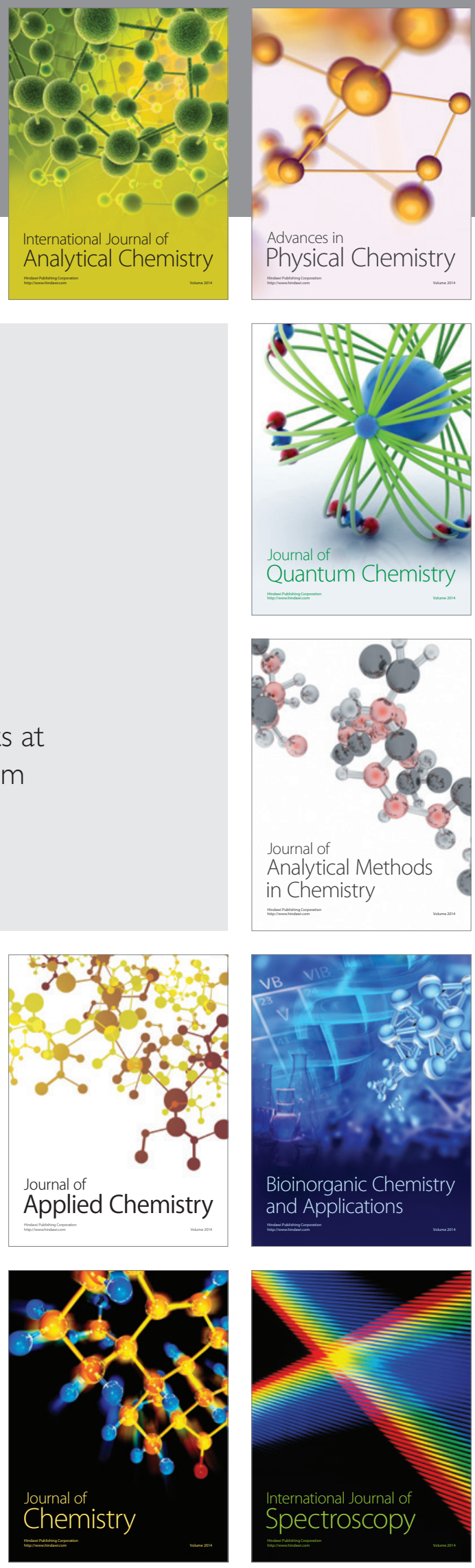